\title{
Intravenous lidocaine Increases the Efficacy of Propofol Anesthesia for Gastroscope Implanted:A Randomised, Double-blind, Controlled Study
}

\section{Fu Shi}

Department of Anesthesiology, Liaocheng People's Hospital Jie Chen

Department of Anesthesiology, Liaocheng People's Hospital

Guang Ren

Department of Anesthesiology, Liaocheng People's Hospital

Rong Du

Department of Anesthesiology, Liaocheng People's Hospital

Xiang Wang ( $\nabla$ wxxlymz@sina.com )

Department of Anesthesiology, Liaocheng People's Hospital

\section{Research Article}

Keywords: Intravenous lidocaine, Propofol, Gastroscopy, The median effective dose

Posted Date: February 9th, 2021

DOI: https://doi.org/10.21203/rs.3.rs-149394/v1

License: (9) This work is licensed under a Creative Commons Attribution 4.0 International License. Read Full License 
Intravenous lidocaine increases the efficacy of propofol anesthesia for gastroscope implanted:a randomised, double-blind, controlled study

Fu Shi ${ }^{1}$, Yan-Jie Chen ${ }^{1}$, Xiang-Rong Du ${ }^{1}$, Chun-Guang Ren ${ }^{1}$,Xu-Xiang Wang ${ }^{1} \Delta$

${ }^{1}$ Department of Anesthesiology, Liaocheng People's Hospital, Liaocheng, Shandong Province 252000, China.

Correspondence:Xu-Xiang Wang, Department of Anesthesiology of Liaocheng People's Hospital, No.67. Dongchang West Road, Liaocheng City, 252000 China. (e-mail:wxxlymz@sina.com).

\begin{abstract}
Backgroud: Gastroscope insertion is a distressing experience and propofol use for sedation can result in inhibition of circulation and respiration. Intravenous (IV) infusion of lidocaine has been shown to reduce propofol requirements during surgery.The goal of this study was to evaluate the impact of IV lidocaine on the median effective dose (ED50) of propofol and other outcomes after surgery.
\end{abstract}

Method: Ninety adult patients undergoing analgesic gastroscopy were randomly divided into two groups ( 45 cases in each). The lidocaine group was slowly injected with $1 \%$ lidocaine at $1.5 \mathrm{mg} / \mathrm{kg}$ while the saline group was given the same volume of saline before propofol induction. Motion and cough reaction were regarded as the judgment index of gastroscope implanted. The dose-response curves were made in the two groups when implanted gastroscope according to the formula of $\mathrm{Y}=\mathrm{Y}_{\min }+\left(\mathrm{Y}_{\max }-\mathrm{Y}_{\min }\right) /\left[1+10^{\log (\mathrm{ED} 50-\mathrm{X})^{*} \mathrm{~m}}\right]$. The primary outcome were the ED50 and requirement of propofol. Secondary outcomes were:intraoperative hemodynamic parameters,adverse reaction, awakening time,post-operative pain and fatigue.

Results: Compared with the group S, the dose-response curve showing percentage of gastroscope implanted after propofol anesthesia in the L group shifted to the left. Intravenous infusion of lidocaine decreased the ED50 of propofol (group S 1.80mg/kg, group L $1.56 \mathrm{mg} / \mathrm{kg}$ ).Compared with group $\mathrm{S}$, induction, additional requirement and 
total dose of propofol anesthesia, and incidence of bucking were significantly reduced in group L $(P<0.05)$. Post-operative fatigue scores in group $\mathrm{L}$ were lower than that in group $\mathrm{S}(P<0.05)$.

Conclusion: Intravenous lidocaine could reduce the consumption of propofol anesthesia and reduce adverse reaction in patients undergoing painless gastroscopy.

Trials registration: ChiCTR2000038542. Data of registration: Sep 23th, 2020.

Keywords: Intravenous lidocaine, Propofol, Gastroscopy, The median effective dose

\section{Background}

At present, painless gastroscopy is the preferred method for the diagnosis of upper gastrointestinal diseases, and satisfactory anesthetic effect is the key to the operation.The purpose of using anesthetic are to relieve discomfort and diminish stress response of the procedure. Propofol is commonly used for painless gastroscopy because it owns the characteristics of short onset time,deep sedation and quick recovery[1].However, application of propofol alone has a high incidence of respiratory depression and circulatory fluctuation. Anesthesiologists have tried to address these adverse reactions by adding other analgesic and sedative adjuncts to propofol anesthesia.Administration of remimazolam could acquire rapid sedation in patients undergoing a diagnostic upper gastrointestinal endoscopy, but it need add the appropriate dosage intermittent because of its quick recovery profile and the pharmacological properties need to be further studied[2].Propofol combined with opioids could provide satisfactory sedative and analgesic effects, but it can increase the risk of hypoxemia and apnea[3]. In endoscopic surgery, dexmedetomidine has antianxiety and sedative effects without respiratory depression, but can cause hypotension and bradycardia[4].

Recently, the intravenous adjuvant use of lidocaine has attracted extensive research interest due to its anti-inflammatory and analgesic effects.Studies showed that intravenous lidocaine can reduce opioid consumption, accelerate the recovery of gastrointestinal function and shorten hospital stay[5, 6]. Also, IV lidocaine infusion with a bolus of $1.5 \mathrm{mg} / \mathrm{kg}$ followed by $2 \mathrm{mg} / \mathrm{kg} / \mathrm{h}$ can significantly attenuate IL-8, 
IL-6 and IL-1ra production and alleviate postoperative pain for laparoscopic cholecystectomy patients[7].

Furthermore, Naris reported that IV lidocaine could reduce the minimum alveolar concentration of sevoflurane in response to a painful stimulation in dogs[8].During open radical prostatectomy, IV lidocaine reduced the end-tidal sevofurane concentration when the bispectral index maintain between 40 and $60[9]$.Forster and colleagues also showed that IV infusion of lidocaine reduced $50 \%$ propofol dose requirements during colonoscopy and improved postoperative fatigue[10].However, whether IV lidocaine can reduce the dose of propofol in patients undergoing gastroscopy implanted has not been investigated.Therefore,we aimed to determine whether intravenous lidocaine could affect the median effective dose (ED50) of propofol for painless gastroscopy. The hypothesis of this study was that IV lidocaine could decrease propofol requirements,decrease adverse reactions and improve other outcomes.

\section{Materials and Methods}

\section{Patients}

The present study was approved by the Ethics Committee of the Liaocheng People's Hospital in Shandong Province, China.The study was carried out according to the guidelines of the Declaration of Helsinki, and registered on 23 September 2020 on the Chinese Clinical Trial Registry (ChiCTR2000038542; main researcher: Fu Shi). The applied clinical study aims to investigate the effect of intravenous lidocaine on anesthetic dosage and postoperative recovery quality in endoscopic surgery. Meanwhile, this investigation of lidocaine for painless gastroscopy is an important part of the registered clinical study. All recruited patients were signed informed consent.This study was carried out between 28 September and 30 November 2020.A total of 90 patients with American Society of Anesthesiologists (ASA) physical status I or II, who were consecutively scheduled for painless gastroscopy diagnosis at the Endoscopy Examination Center of the Liaocheng People's Hospital, were enrolled in the trail. They were randomly divided into two groups by using a computer to 
generate random number list at a 1:1 ratio(45 patients in each group). Put the patient's group number(group L and group S) in the envelope.Anesthesia assistant prepared two same syringes, a syringe containing 1\% lidocaine and another containing the same volume saline.After the patient entered the room, another anesthesiologist who was blind to the group allocation operated the anesthesia for the surgery.Patients who were age $<18$ and $>60$ years, weighed $<45 \mathrm{~kg}$ or $>100 \mathrm{~kg}$,with severe cardiac, pulmonary, hepatic or renal dysfunction, with a history of opioid and sedative drug abuse,allergic to lidocaine were excluded.Additionally, we excluded patients if they refuse to sign the informed consent.

\section{Anesthesia and Intervention}

After entering the examination room, the patient was placed on the bed on the left side, and was given pure oxygen $(3 \mathrm{~L} / \mathrm{min})$ via a nasal tube.Electrocardiogram (ECG), pulse,noninvasive arterial blood pressure(NBP), and oxygen saturation $\left(\mathrm{SpO}_{2}\right)$ were monitored.Patients in group L were administered intravenously a bolus dose of $1 \%$ lidocaine $(1.5 \mathrm{mg} / \mathrm{kg})$ and the same volume saline were given in the group $\mathrm{S}$ before propofol anesthesia induction. Then, the patient was repeatedly injected with propofol at an incremental dose, first at $1.2 \mathrm{mg} / \mathrm{kg}$ and then increased by $0.2 \mathrm{mg} / \mathrm{kg}$ at $15 \mathrm{~s}$ intervals. The propofol dose that gastroscopy was implanted without motion and cough reaction was recorded.The dose-response curve was matched using the formula of $Y=Y_{\min }+\left(Y_{\max }-Y_{\min }\right) /\left[1+10^{\log (E D 50-X)^{*} \mathrm{~m}}\right]$. Meanwhile, if motor responses occurred, the compensate dose of propofol was intravenous injected at $0.5 \mathrm{mg} / \mathrm{kg}$ in order to meet operation needs. If hypotension (systolic blood pressure $<90 \mathrm{mmHg}$ or descending $20 \%$ basal value) occurred, $6 \mathrm{mg}$ ephedrine was intravenous injected. If bradycardia ( $\mathrm{HR}<50 \mathrm{bpm}$ ) occurred, $0.2 \mathrm{mg}$ atropine was intravenous injected. If the $\mathrm{SpO}_{2}$ was $<90 \%$ for $\geq 10 \mathrm{sec}$, anesthetist lift the chin quickly. The apnea was defined as no respiratory motility $\geq 10$ sec. If apnea occurred, the patients were given positive pressure-assisted ventilation by the mask. After operation, the patient was transferred to postanesthesia care unit. 


\section{Measurements}

The primary outcome were the ED50 of propofol for the gastroscope implanted and additional, total propofol consumption during the surgery. Secondary outcomes were: changes in the $\mathrm{HR}, \mathrm{NBP}$ and $\mathrm{SpO}_{2}$ during the surgery at the following time points: before induction (T0); after anesthesia induction but before the procedure (T1); after endoscope implantation (T2); after endoscope removal (T3); adverse reaction:the number of apnea episodes(defined as no abdominal breathing motion $\geq 10 \mathrm{~s}$ ), the number of hypotension, the number of bucking and motion.Additional secondary endpoints were:awakening time (set as the time from the end of surgery to eye opening time); the post-surgery pain and fatigue score (assessed on the $0-10 \mathrm{~cm}$ visual analog scale VAS; the higher the score, the more intense the pain and exhaustion).

\section{Statistical Analyses}

We estimated a pilot study size of 20 patients that the propofol dose for the gastroscope implanted was $109.8 \pm 36.2$ in group $\mathrm{S}$ and $131.2 \pm 42.6$ in group L. The significance was set at 0.05 and power was set at $80 \%$. The calculated results were 43 patients in each group undertaken by PASS15.0 (NCSS,Kaysville,UT,USA). Finally, a total of 90 patients were included.

The Kolmogorov-Smirnov test was used to analyze the normally distribution of data. Measurement data are expressed as the mean \pm standard deviation $(\overline{\mathrm{x}} \pm \mathrm{s})$ and were compared using the unpaired Student's t tests.Categorical variables are presented as percentage of numbers (\%) and were compared using the $\chi 2$ test or Chi-square test. All experimental data were performed using SPSS version 16 software (SPSS Inc., Chicago, IL) and $P<0.05$ was considered statistically significant.

The dose-response curve of gastroscopy implanted under propofol anesthesia was drawn using the GraphPad Prism software 5.0 (GraphPad Prism, Inc., San Diego, CA)[11].The Prism equation $Y=Y_{\min }+\left(Y_{\max }-Y_{\min }\right) /\left[1+10^{\log (E D 50-X) * m}\right]$ : where $Y$ is the percentage of gastroscopy implanted successful, $\mathrm{X}$ is the logarithmic drug dose, $\mathrm{m}$ describes the steepness of the family of curves; and ED50 is the propofol dose that produces a half-maximal effect. 


\section{Results}

\section{The design diagram of the study and clinical data in the two groups}

First, a total of 105 patients were recruited for the study. Of these, three patients had a severe hypertension, two patients suffered alcohol dependence syndrome,and ten patients refused to sign the informed consent.Finally, 90 patients (45 in each group) participated in the research(Figure 1).The characteristics of patients in the two groups are presented in Table 1.There were no significant differences between the groups in terms of age, gender, American Society of Anesthesiology (ASA) class, weight, or duration of operation.

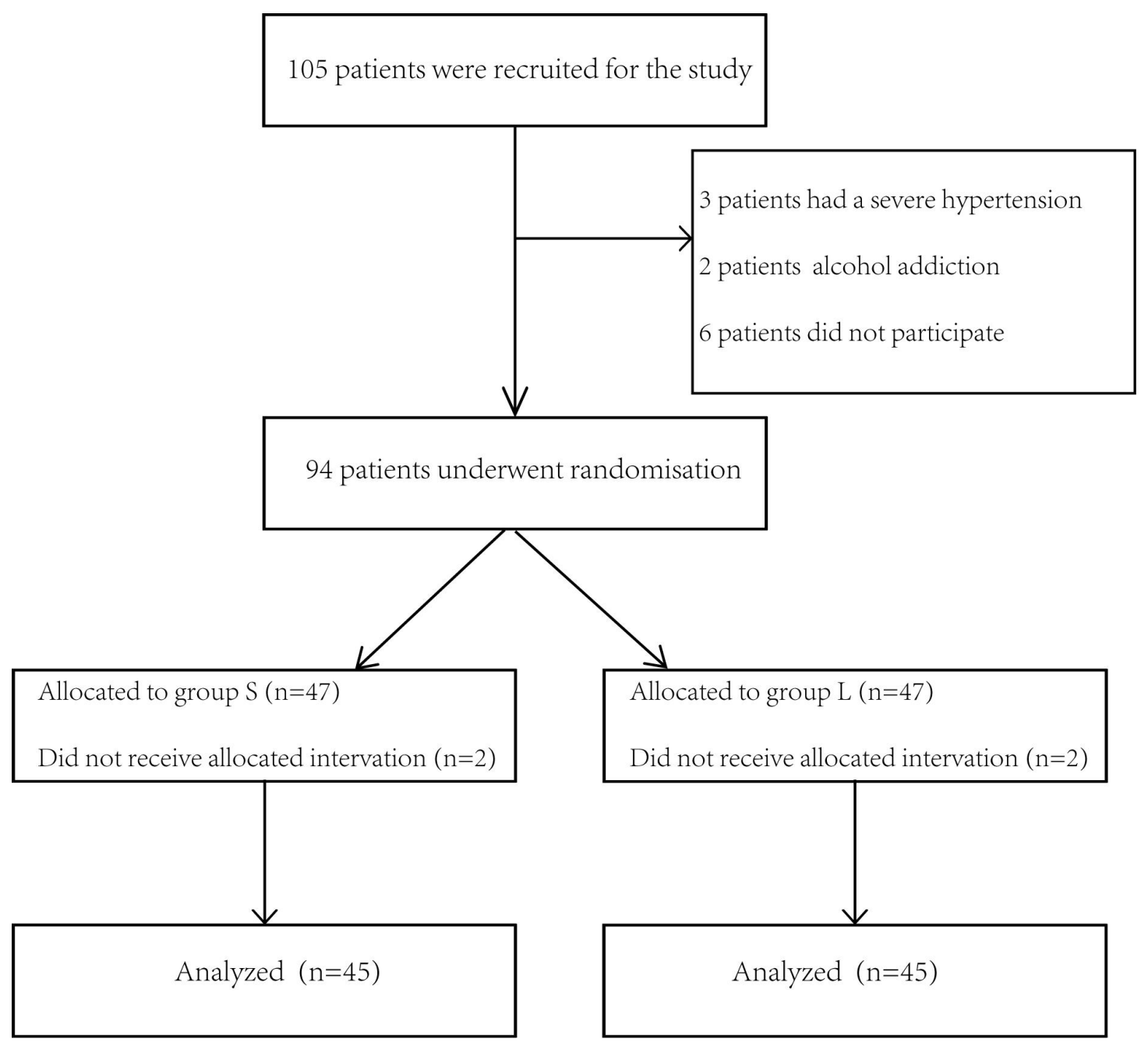

Fig. 1 study population flow diagram 
Table 1 Comparison of general data of patients in the two groups $(\mathrm{n}=45)$

\begin{tabular}{|c|c|c|c|c|}
\hline Variables & Saline $(n=45)$ & Lidocaine $(n=45)$ & $t / \chi^{2}$ & $P$ value \\
\hline Gender (F/M) & $24 / 21$ & $26 / 19$ & 0.180 & 0.617 \\
\hline Age $(y r)$ & $50.44 \pm 7.15$ & $50.18 \pm 9.44$ & 0.151 & 0.880 \\
\hline Weight $(\mathrm{kg})$ & $65.49 \pm 8.66$ & $68.98 \pm 9.18$ & 1.855 & 0.067 \\
\hline $\operatorname{ASA}(\mathrm{I} / \mathrm{II})$ & $24 / 21$ & $23 / 22$ & 0.045 & 0.833 \\
\hline Duration of surgery $(\mathrm{s})$ & $325.70 \pm 17.87$ & $328.60 \pm 18.16$ & 0.749 & 0.456 \\
\hline
\end{tabular}

Notes: Data are presented as mean \pm SD or numbers (\%). There were no significant differences in both groups $(P>0.05)$.

\section{Intravenous infusion of lidocaine reduced the propofol requirement}

Intravenous infusion lidocaine moved the dose-response curve to the left compared with the saline group (Figure 2A). The ED50 of propofol in group $\mathrm{L}(1.56 \mathrm{mg} / \mathrm{kg}, 95 \% \mathrm{CI}: 1.53 \sim 1.58 \mathrm{mg} / \mathrm{kg})$ was lower than that in group $\mathrm{S}$ (1.80mg/kg ,95\%CI: $1.79 \sim 1.83 \mathrm{mg} / \mathrm{kg})$.Compared with group S (125.50 $24.06 \mathrm{mg})$, the induction dose of propofol anesthesia for surgery was reduced in group L (112.70 $\pm 16.20 \mathrm{mg}, t=2.958, P<0.01)$. During the surgery, the additional dose of propofol in group L was lower than that in group S (group S:79.71 $\pm 9.11 \mathrm{mg}$; group L: $65.42 \pm$ $10.57 \mathrm{mg}, t=6.870, P<0.01)$. The total consumption dose of propofol during the surgery was less in the group $\mathrm{L}(178.10 \pm 20.27 \mathrm{mg})$ than that in group $\mathrm{S}(205.20 \pm$ $27.81 \mathrm{mg}, \mathrm{n}=45$ for both $, t=5.278, P<0.01$ ) (Figure $2 \mathrm{~B}$ ).
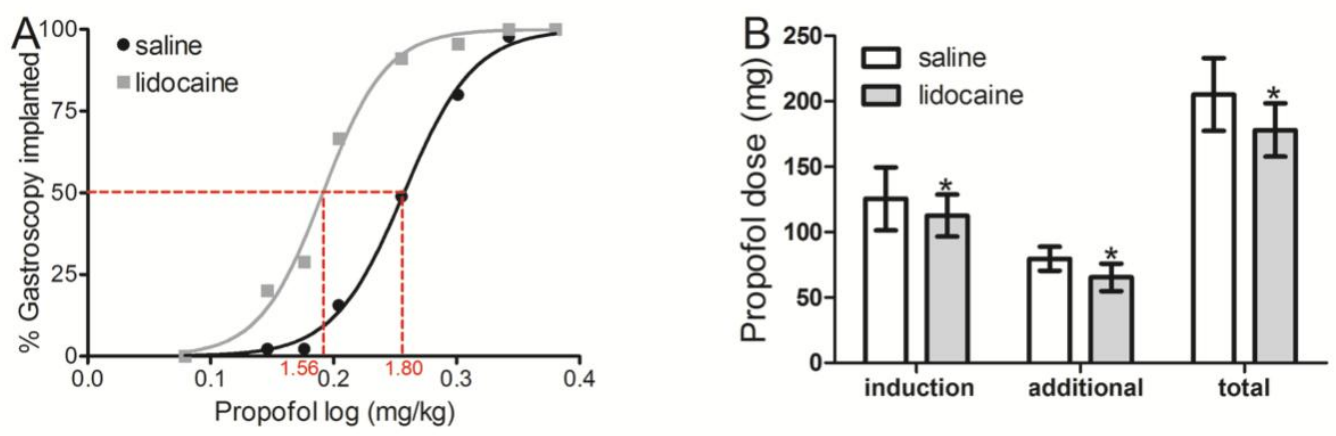

Fig. 2 A:The dose-response curves of gastroscopy implanted under propofol anesthsia $\mathrm{X}$ axle shown the log-converted drug dose using the Prism 5 software, $\mathrm{Y}$ axle shown the percentage of 
gastroscope implanted at different dose of propofol anesthesia. B: the induction, additional and total consumption dose of propofol in the two groups.

\section{IV lidocaine decreased the fluctuation of HR and MAP when the endoscope implanted}

The differences in mean arterial pressure(MAP) and heart rate(HR) between the two groups at T0,T1 and T3 were not statistically different.During the endoscope implantation, the MAP (group S:87.80 \pm 7.66 ; group L: $83.69 \pm 9.17, P<0.05$ ) and HR (group S:84.98 \pm 7.01 ; group L: $81.04 \pm 7.47, P<0.05$ ) were significantly higher in group S than in group L at T3(Figure3).
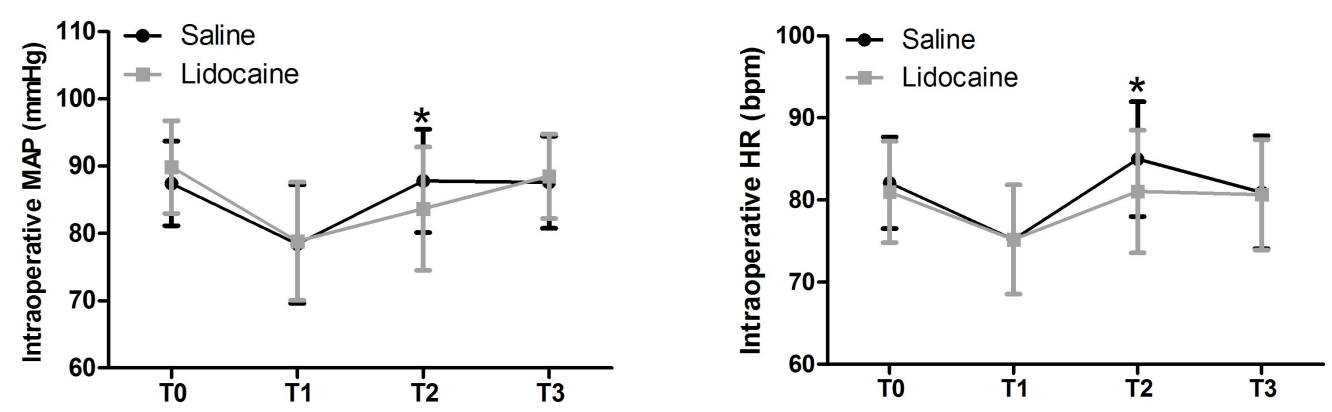

Fig. 3 Comparison of MAP and HR at different time points in the two groups

Notes: $\mathrm{T} 0=$ before induction $; \mathrm{T} 1=$ after anesthesia induction $; \mathrm{T} 2=$ after endoscope implantation; T3 =after endoscope removal. ${ }^{*} P<0.05$,compared with saline group.

\section{IV lidocaine decreased the incidence of bucking response during surgery}

The incidence of bucking response was significantly higher in group $\mathrm{S}$ than in group

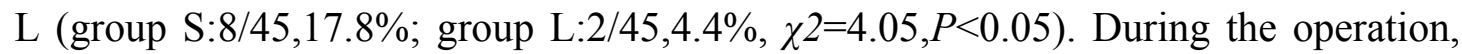
the incidence of motion, apnea and hypotension occurred at a high frequency in group $\mathrm{S}$ that in group L, but were not statistically significant.The severe apnea and hypoxemia were not occurred in the two groups(Figure4). 

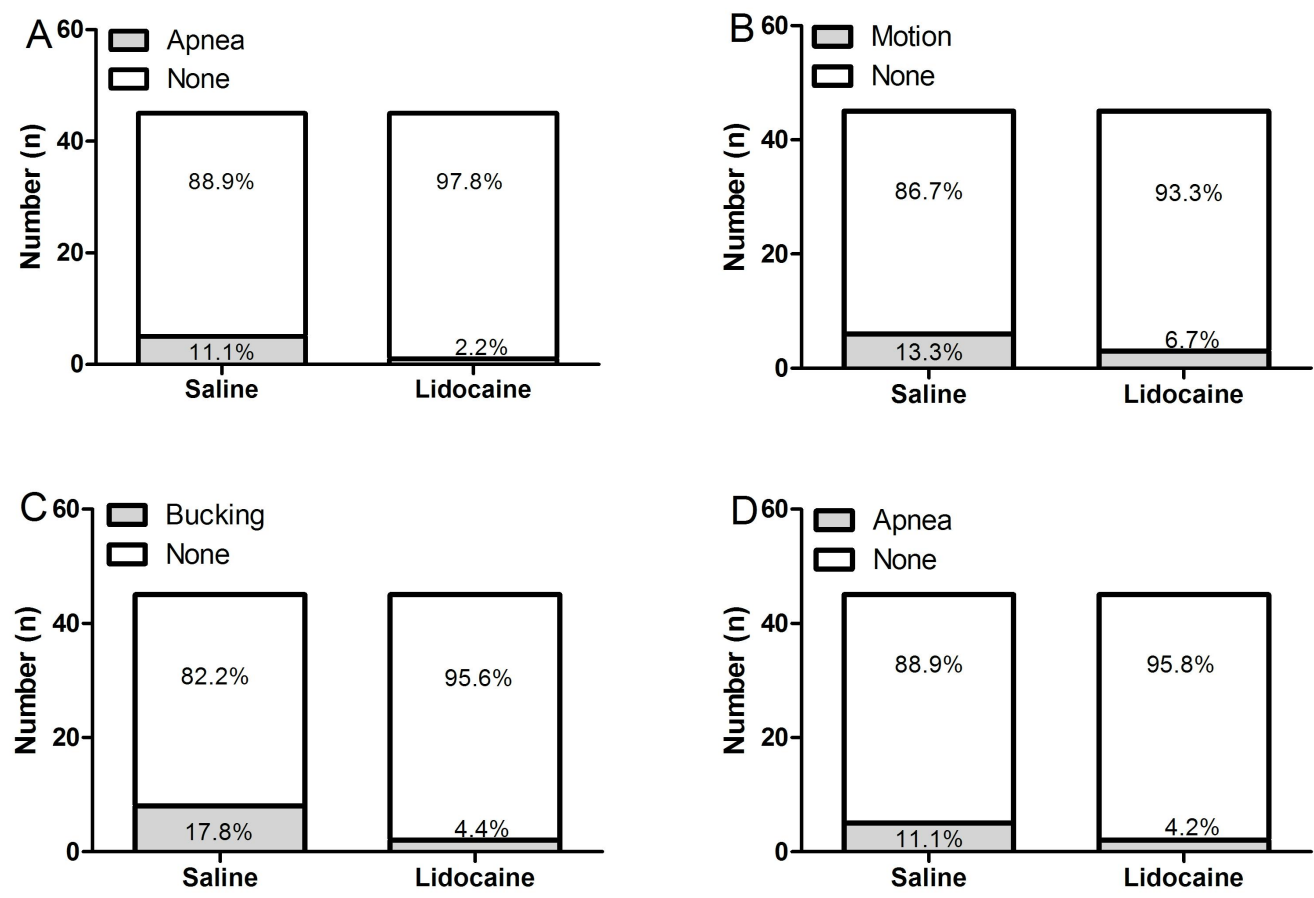

Fig. 4 Comparison of the adverse reaction between the two groups during the surgery

Note: Grey color shown the percentage of adverse reaction in the two groups.

The pain score and recovery time were not affected by intravenous infusion

\section{lidocaine}

The fatigue score after surgery was lower in group L $(1.87 \pm 1.21)$ than that in group $\mathrm{S}(2.93 \pm 1.50, \mathrm{t}=3.706, P<0.05)$. Compared with group $\mathrm{S}$, group $\mathrm{L}$ did not show a significant difference in the recovery time and VAS score after the surgery(Figure5).
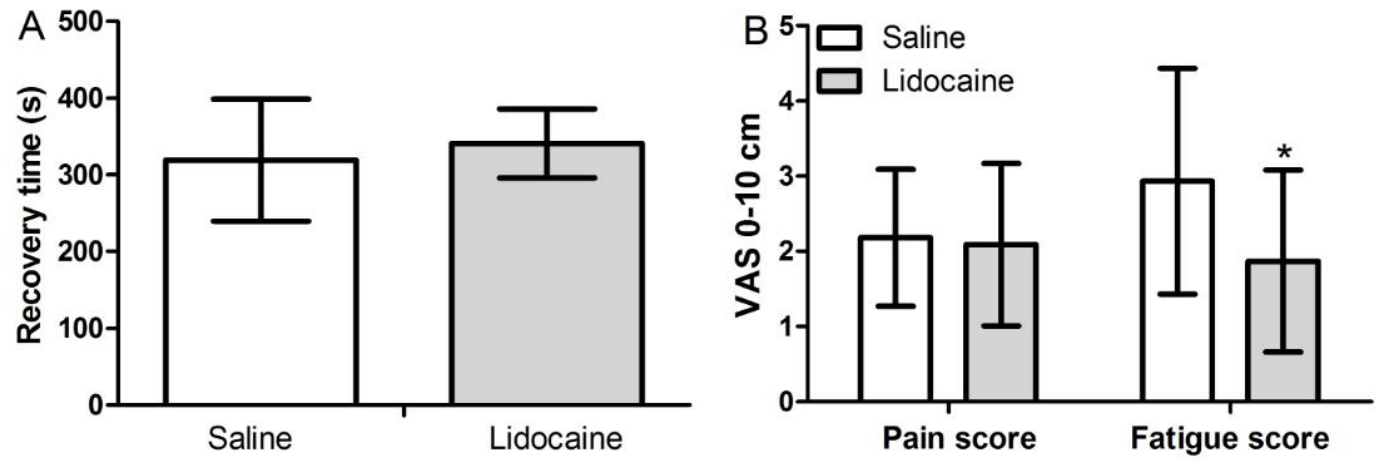

Fig. 5 Comparison of the recovery time, pain and fatigue score in the two groups ${ }^{*} P<0.05$; compared with saline group 


\section{Discussion}

This study revealed that intravenous infusion of lidocaine could decrease the ED50 of propofol and reduce the induction, additional and total dose of propofol anesthesia for surgery. Also, IV lidocaine can alleviate the fluctuation of HR and MAP and bucking response during the gastroscope implantation. Furthermore, IV lidocaine could also improve the post-operation satisfaction of patients, but not affect the recovery time and pain scores.Moreover, the symptoms of lidocaine toxicity (tinnitus, perioral numbness, blurred vision and arrhythmia) were not found after recovery from surgery.

Gastroscope diagnosis technology is currently the most important method to diagnose digestive tract diseases, but patients need to endure great pain and psychological pressure[12]. Propofol own the rapid action, deep sedation, predictable recovery pharmacological properties and could eliminate the discomfort caused by mechanical stimulation[13]. However, a bolus of intravenous propofol can also increase the incidence of respiratory depression and low blood pressure[14]. Recent experimental study demonstrated that perioperative lidocaine infusion could bring many clinical benefits for patients, such as decrease the sedative drug dose, alleviate visceral pain and enhance the gastrointestinal function recovery[15]. Therefore, we investigated the effect of IV lidocaine on propofol anesthesia during the gastroscope implantation.

A basic study suggested that intravenous lidocaine can exert anaesthetic-sparing effect and decrease the minimum alveolar concentration of isoflurane in horses[16]. A clinical study demonstrated that intravenous lidocaine could significantly reduce total propofol consumption and decrease the prevalence of apnea episodes during painless colonoscopy[17]. Another clinical findings also showed that IV lidocaine can significant reduce overall propofol dose in the elderly during colonoscopy[18].Indeed, our study support previous work showing that IV lidocaine turns the dose-response curve to the left and decreases the propofol induction requirements for surgery. Propofol acts on gamma-aminobutyric acid $\left(\mathrm{GABA}_{\mathrm{A}}\right)$ receptors, increasing the 
permeability of cell membranes to chloride ions and producing inhibitory postsynaptic potentials[19]. Nordmark and colleagues have shown that lidocaine can block the re-uptake of GABA in the central nervous system and increase the influx of chloride ions, thereby reducing the excitability of neurons[20]. These findings further showed that the propofol-sparing effects of lidocaine might be related to the interactions inhibition of central excitatory transmission between propofol and lidocaine.

The strong mechanical irritation to the throat can excite the sympathetic nervous system, resulting in a cardiovascular response.In our study, a single application of propofol can also be used to complete the operation. However, the fluctuations of MAP and HR after propofol induction were significantly. Lidocaine is a local anesthetic that can be given intravenously. It was reported that intravenous infusion of lidocaine could blunt the hemodynamic response to endotracheal intubation during induction of anaesthesia[21]. Hypotension is a common complication after induction of general anaesthesia and lidocaine-ketamine combination could significant decrease incidence of hypotension compared to ketamine when used for endotracheal intubation in patients with circulatory failure[22].Our study found that the fluctuation of MAP and HR after gastroscopy implanted in group L were lower than those in group S. In addition, IV lidocaine can reduce the incidence of cough in patients undergoing gastroscopy. A meta-analysis has shown that intravenous lidocaine may be a best chose to decrease emergence coughing during anesthesia recovery and endotracheal tube pulled out in adult elective surgery[23].Furthermore, IV lidocaine may exert central anti-nociceptive effects by action on muscarinic and nicotinic receptors, which reinforce the inhibitory descending pain pathway in rats experiment[24].These findings in our study showed that lidocaine could stabilize the hemodynamic fluctuation after gastroscopy implanted, and reduce the incidence of intraoperative cough. The reason may be that IV lidocaine could inhibit the afferent and efferent pathways of pain reflex, so as to reduce the sympathetic reflex caused by strong stimulus to the throat during gastroscopy.

The incidence of apnea between the two groups was 
not statistically significant. However, our study shown that apnea occurred at a high frequency in group $\mathrm{S}$ than that in group L (group S:5/45,11.1\%; group L:1/45,2.2\%, $P>0.05$ ) and IV lidocaine improved respiratory depression to a certain extent. Excessive use of propofol can lead to respiratory depression. Lidocaine was regarded as an adjunct to propofol anesthesia and played the sedative-sparing effect[18]. Intravenous lidocaine decreasing the propofol requirement for painless gastroscopy may account for the this observation. Erb, $\mathrm{T} O \mathrm{O}$ and colleagues reported that the incidence of laryngospasm evoked by laryngeal stimulation was reduced from $38 \%$ to $15 \% 2 \mathrm{~min}$ and to $18 \% 10 \mathrm{~min}$ after IV $2 \mathrm{mg} / \mathrm{kg}$ lidocaine[25].Although the effect was short-lived, it can cover the procedure of gastroscopy. The explanation for it was that lidocaine dilates bronchial smooth muscle and then ensured upper respiratory tract unobstructed.

Aeration and mechanical stimulation of the gastrointestinal tract can lead to visceral pain. In a study by $\mathrm{Su}$ Liu et al, it shown that IV lidocaine can decrease the 1-min VAS score after colonoscopy[17]. Furthermore, lidocaine was used as an intravenous adjunct to anesthesia, it could decrease postoperative pain and shorten the time to the first flatus and bowel sounds after laparoscopic colon surgery[26]. These evidence indicated that lidocaine played an important role in analgesic effect and resumption of bowel function.Our study demonstrated that pain scores in the lidocaine group were lower than that in saline group, but was not statistically significant.The time of gastroscopy is shorter than that of colonoscopy and the short periods of mechanical stimulation does not cause significant discomfort for patients. It could be that analgesic effect of lidocaine has not shown in painless gastroscopy. Moreover, IV lidocaine can alleviate the fatigue in patients after operation and it may be related to the propofol-sparing effect.

Our study also have a few limitations.First, we did not measure the depth of propofol anesthesia after lidocaine administration. We mainly depended on clinical index accounting for the anesthetic sparing effect of lidocaine. If conditions permit, we can use the bispectral index (BIS) to monitor the anesthesia depth. Then it could better explain if lidocaine affect the cortex activity. Second, lidocaine did not affect 
the postoperative awakening time of the patients, and it may be that the tapping and calling by the medical staff in the recovery room affected the awakening process of anesthesia. Third, Lidocaine as an amide local anesthetic is intravenous injected, but the accompanying toxic and side effects of local anesthetics, such as vertigo, tinnitus, headache, visual impairment, convulsions and arrhythmias, may bring potential safety risks. Greenwood et al. verified the safety of administration dose by measuring the level of lidocaine in the blood[27]. Patients undergoing colorectal cancer surgery were intravenously injected with $1.5 \mathrm{mg} / \mathrm{kg}$ lidocaine during anesthesia induction. Patients with less than $70 \mathrm{~kg}$ were continuously injected with $2 \%$ lidocaine at $3 \mathrm{mg} / \mathrm{h}$, and patients with more than $70 \mathrm{~kg}$ were continuously injected with $6 \mathrm{mg} / \mathrm{h}$. The results showed that the average total serum lidocaine concentrations of $4.05 \mu \mathrm{g} / \mathrm{ml}$.Therefore, in our study, $1.5 \mathrm{mg} / \mathrm{kg}$ of $1 \%$ lidocaine was injected intravenously before anesthesia induction, and the cardiovascular toxicity or any symptoms of toxicity were not observed in our patients.

\section{Conclusions}

Intravenous infusion of lidocaine could enhance propofol anesthesia for painless gastroscopy. Lidocaine also stabilizes the hemodynamic fluctuation and reduces the incidence of intraoperative cough.Our study provides a new evidence for the use of lidocaine adjunct to propofol anesthesia in painless gastroscopy.However, multi-center and large sample studies are still needed for verification.

\section{Abbreviations}

ED50:the median effective dose;MAP:mean noninvasive arterial blood pressure; $\mathrm{SPO}_{2}$ :oxygen saturation;HR:heart rate;IV lidocaine:Intravenous lidocaine

\section{Acknowledgments}

Not applicable.

\section{Author's contributions}

Xuxiang Wang provided guidance for experimental design. Yanjie Chen and Xiangrong Du performed the clinical tests. Fu Shi Competed data analysis and write the manuscript. All authors read and approved the final manuscript. Finally, we thank 
Chunguang Ren very much for revising the manuscript.

Funding No funding was received for this study.

\section{Availability of data and materials}

The datasets generated and analyzed during the current study are available from the corresponding author on reasonable request.

\section{Ethics approval and consent to participate}

The present study acquired the Chinese Clinical Trial Registry (ChiCTR2000038542) and was approved by the Ethics Committee of the Liaocheng People's Hospital in Shandong Province, China. Written informed consent was obtained from the guardians of all the patients included in this study.

\section{Consent for publication}

All authors declare consent for publication.

\section{Competing interests}

All authors have no potential conflicts of interest to disclose.

\section{Author details}

${ }^{1}$ Department of Anesthesiology, Liaocheng People's Hospital, No.67. Dongchang

West Road, Liaocheng City,Shandong Province 252000,China.

\section{References}

1. Li DN, Zhao GQ, Su ZB: Propofol Target-controlled Infusion in Anesthesia Induction during Painless Gastroscopy. J Coll Physicians Surg Pak 2019, 29(7):604-607.

2. Borkett KM, Riff DS, Schwartz HI, Winkle PJ, Pambianco DJ, Lees JP, Wilhelm-Ogunbiyi K: A Phase IIa, randomized, double-blind study of remimazolam (CNS 7056) versus midazolam for sedation in upper gastrointestinal endoscopy. Anesth Analg 2015, 120(4):771-780.

3. Nieuwenhuijs DJ, Olofsen E, Romberg RR, Sarton E, Ward D, Engbers F, Vuyk J, Mooren R, Teppema LJ, Dahan A: Response surface modeling of remifentanil-propofol interaction on cardiorespiratory control and bispectral index. Anesthesiology 2003, 98(2):312-322.

4. Eberl S, Preckel B, Bergman JJ, van Dieren S, Hollmann MW: Satisfaction and safety using dexmedetomidine or propofol sedation during endoscopic oesophageal procedures: A randomised controlled trial. Eur J Anaesthesiol 2016, 33(9):631-637.

5. Dunn LK, Durieux ME: Perioperative Use of Intravenous Lidocaine. Anesthesiology 2017, 126(4):729-737.

6. Wang T, Liu H, Sun JH, Wang L, Zhang JY: Efficacy of intravenous lidocaine in improving post-operative nausea, vomiting and early recovery after laparoscopic gynaecological surgery. Exp Ther Med 2019, 17(6):4723-4729. 
7. Song X, Sun Y, Zhang X, Li T, Yang B: Effect of perioperative intravenous lidocaine infusion on postoperative recovery following laparoscopic Cholecystectomy-A randomized controlled trial. Int J Surg 2017, 45:8-13.

8. Thengchaisri N, Mahidol C: Evaluating the effects of continuous intravenous infusions of tramadol and tramadol-lidocaine on sevoflurane minimum alveolar concentration (MAC) and entropy values in dogs. J Vet Med Sci 2019, 81(5):682-688.

9. Weinberg L, Jang J, Rachbuch C, Tan C, Hu R, McNicol L: The effects of intravenous lignocaine on depth of anaesthesia and intraoperative haemodynamics during open radical prostatectomy. BMC Res Notes 2017, 10(1):248.

10. Forster C, Vanhaudenhuyse A, Gast P, Louis E, Hick G, Brichant JF, Joris J: Intravenous infusion of lidocaine significantly reduces propofol dose for colonoscopy: a randomised placebo-controlled study. Br J Anaesth 2018, 121(5):1059-1064.

11. Luo T, Leung LS: Involvement of tuberomamillary histaminergic neurons in isoflurane anesthesia. Anesthesiology 2011, 115(1):36-43.

12. Present situation and future prospects on endoscopic diagnosis and treatment in Asia. Abstracts of the 3rd International Gastrointestinal Consensus Symposium (IGICS). February 20, 2010. Fukuoka, Japan. Digestion 2010, 81(3):152-170.

13. Nishizawa T, Suzuki H: Propofol for gastrointestinal endoscopy. United European Gastroenterol J 2018, 6(6):801-805.

14. Wadhwa V, Issa D, Garg S, Lopez R, Sanaka MR, Vargo JJ: Similar Risk of Cardiopulmonary Adverse Events Between Propofol and Traditional Anesthesia for Gastrointestinal Endoscopy: A Systematic Review and Meta-analysis. Clin Gastroenterol Hepatol 2017, 15(2):194-206.

15. Beaussier M, Delbos A, Maurice-Szamburski A, Ecoffey C, Mercadal L: Perioperative Use of Intravenous Lidocaine. Drugs 2018, 78(12):1229-1246.

16. Villalba M, Santiago I, Gomez de Segura IA: Effects of constant rate infusion of lidocaine and ketamine, with or without morphine, on isoflurane MAC in horses. Equine Vet $\mathrm{J} 2011$, 43(6):721-726.

17. Li X, Lv X, Jiang Z, Nie X, Wang X, Li T, Zhang L, Liu S: Application of Intravenous Lidocaine in Obese Patients Undergoing Painless Colonoscopy: A Prospective, Randomized, Double-Blind, Controlled Study. Drug Des Devel Ther 2020, 14:3509-3518.

18. Chen M, Lu Y, Liu H, Fu Q, Li J, Wu J, Shangguan W: The propofol-sparing effect of intravenous lidocaine in elderly patients undergoing colonoscopy: a randomized, double-blinded, controlled study. BMC Anesthesiol 2020, 20(1):132.

19. D'Antuono M, Louvel J, Kohling R, Mattia D, Bernasconi A, Olivier A, Turak B, Devaux A, Pumain R, Avoli M: GABAA receptor-dependent synchronization leads to ictogenesis in the human dysplastic cortex. Brain 2004, 127(Pt 7):1626-1640.

20. Nordmark J, Rydqvist B: Local anaesthetics potentiate GABA-mediated Cl- currents by inhibiting GABA uptake. Neuroreport 1997, 8(2):465-468.

21. Zou Y, Kong G, Wei L, Ling Y, Tang Y, Zhang L, Huang Q: The effect of intravenous lidocaine on hemodynamic response to endotracheal intubation during sufentanil-based induction of anaesthesia. Anaesthesiol Intensive Ther 2020, 52(4):287-291.

22. Fathy S, Hasanin A, Mostafa M, Ramzy E, Sarhan K, Almenesey T, Safina AG, Hosny O, Hamden GA, Gado AA et al: The benefit of adding lidocaine to ketamine during rapid 
sequence endotracheal intubation in patients with septic shock: A randomised controlled trial. Anaesth Crit Care Pain Med 2020.

23. Tung A, Fergusson NA, Ng N, Hu V, Dormuth C, Griesdale DGE: Pharmacological methods for reducing coughing on emergence from elective surgery after general anesthesia with endotracheal intubation: protocol for a systematic review of common medications and network meta-analysis. Syst Rev 2019, 8(1):32.

24. Abelson KS, Hoglund AU: Intravenously administered lidocaine in therapeutic doses increases the intraspinal release of acetylcholine in rats. Neurosci Lett 2002, 317(2):93-96.

25. Erb TO, von Ungern-Sternberg BS, Keller K, Frei FJ: The effect of intravenous lidocaine on laryngeal and respiratory reflex responses in anaesthetised children*. Anaesthesia 2013, 68(1):13-20.

26. Ahn E, Kang H, Choi GJ, Park YH, Yang SY, Kim BG, Choi SW: Intravenous lidocaine for effective pain relief after a laparoscopic colectomy: a prospective, randomized, double-blind, placebo-controlled study. Int Surg 2015, 100(3):394-401.

27. Greenwood E, Nimmo S, Paterson H, Homer N, Foo I: Intravenous lidocaine infusion as a component of multimodal analgesia for colorectal surgery-measurement of plasma levels. Perioper Med (Lond) 2019, 8:1. 


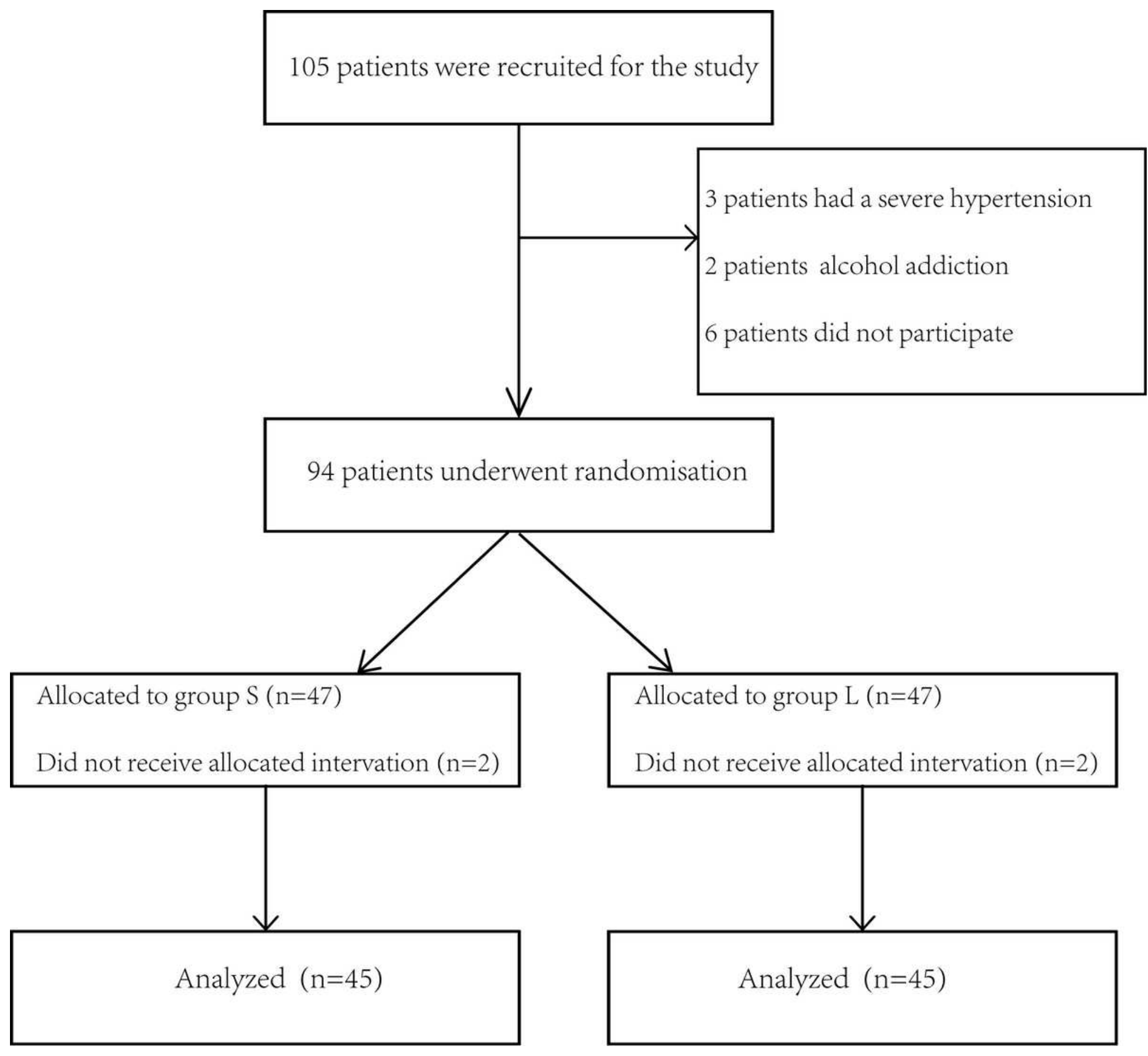

Figure 1

study population flow diagram 

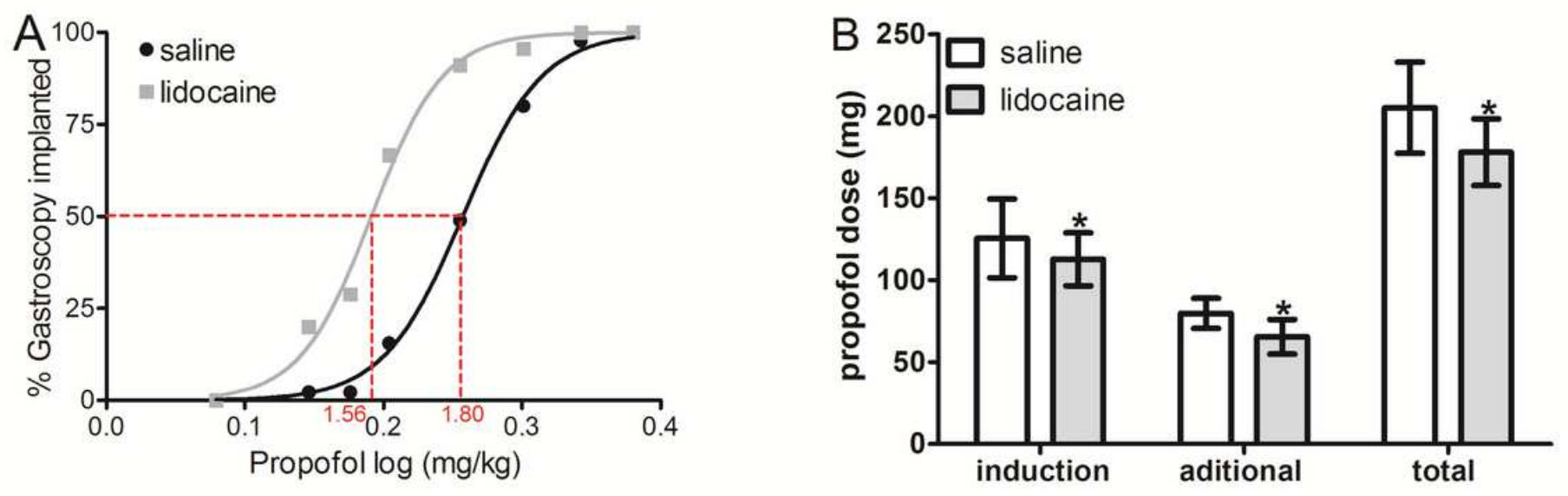

Figure 2

A:The dose-response curves of gastroscopy implanted under propofol anesthsia $X$ axle shown the logconverted drug dose using the Prism 5 software, $\mathrm{Y}$ axle shown the percentage of gastroscope implanted at different dose of propofol anesthesia. B: the induction, additional and total consumption dose of propofol in the two groups.
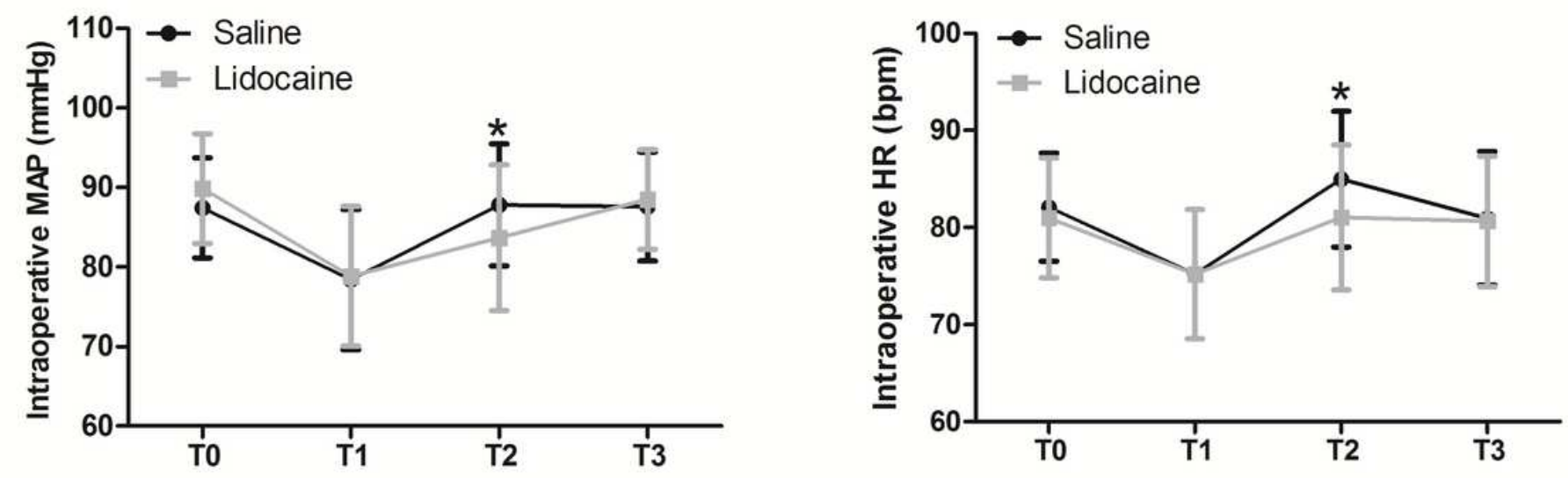

Figure 3

Comparison of MAP and HR at different time points in the two groups Notes: $\mathrm{T} 0=$ before induction ; $\mathrm{T} 1=$ after anesthesia induction; T2=after endoscope implantation; T3 =after endoscope removal. * $\mathrm{P} \otimes$ 0.05 ,compared with saline group 

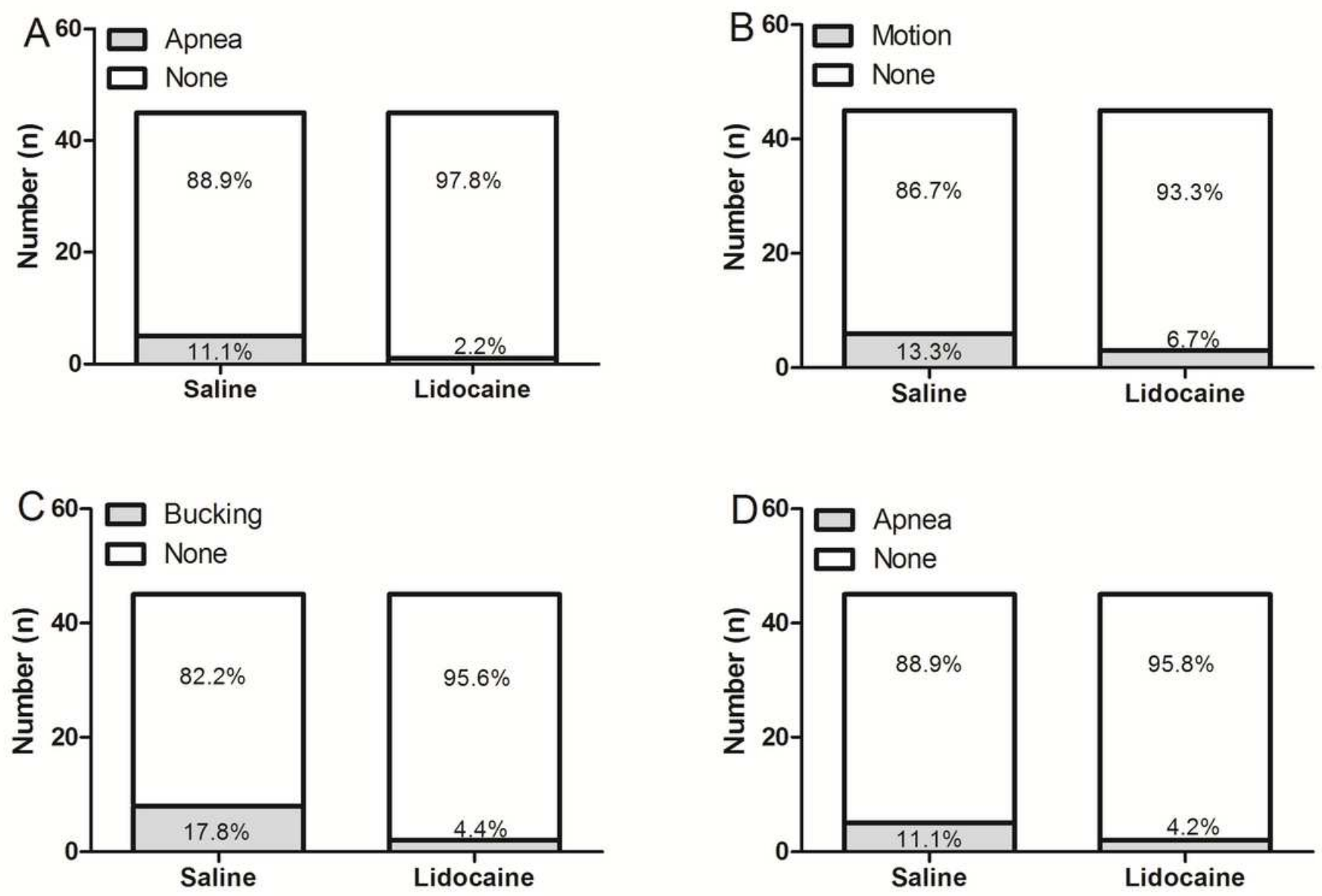

Figure 4

Comparison of the adverse reaction between the two groups during the surgery Note: Grey color shown the percentage of adverse reaction in the two groups.
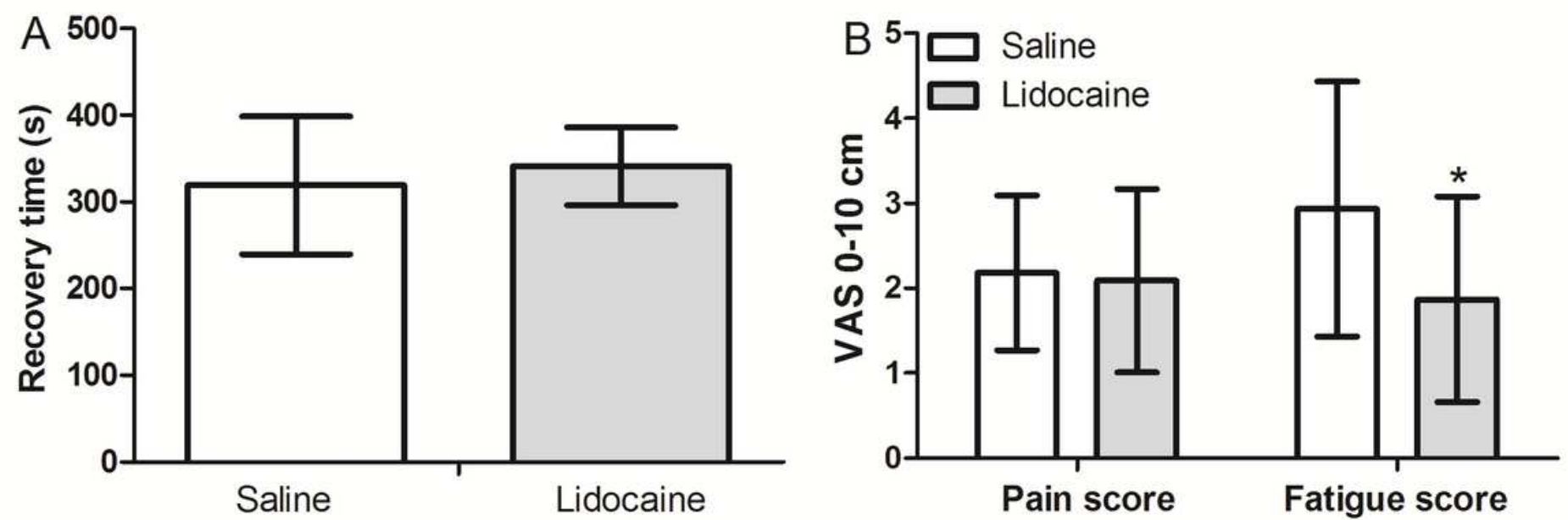

Figure 5 
Comparison of the recovery time, pain and fatigue score in the two groups * $P \otimes 0.05$; compared with saline group

\section{Supplementary Files}

This is a list of supplementary files associated with this preprint. Click to download.

- database.xls

- CONSORT2010Checklist.doc

- researchprotocol.doc 